\title{
Supplementary materials: High-dimensional confounding adjustment using continuous spike and slab priors
}

\author{
Joseph Antonelli* ${ }^{*}$ Giovanni Parmigiani ${ }^{\ddagger}$ and Francesca Dominici ${ }^{\dagger}$
}

\section{Posterior computation}

The laplace distribution has the following representation as a scale mixture of Gaussians with an exponential mixing weight.

$$
\frac{\lambda}{2} e^{-\lambda|\beta|}=\int_{0}^{\infty} \frac{1}{\sqrt{2 \pi \tau^{2}}} e^{-\beta^{2} / 2 \tau^{2}} \frac{\lambda^{2}}{2} e^{-\lambda^{2} \tau^{2} / 2} .
$$

This makes the conditional distribution of $\boldsymbol{\beta}$ multivariate normal. To simplify notation we will also define $\boldsymbol{X}^{*}=\left[\mathbf{1}^{\prime}, \boldsymbol{T}^{\prime}, \boldsymbol{X}\right]$ and $\boldsymbol{\beta}^{*}=\left[\beta_{0}, \beta_{t}, \boldsymbol{\beta}\right]$. Conditional on a value of $\lambda_{0}$ the algorithm iterates through the following steps:

1. Sample $\boldsymbol{\beta}^{*}$ from the full conditional:

$$
\boldsymbol{\beta}^{*} \mid \bullet \sim \operatorname{Normal}\left(\left(\boldsymbol{X}^{* T} \boldsymbol{X}^{*}+\boldsymbol{D}_{\boldsymbol{\tau}}^{-1}\right)^{-1} \boldsymbol{X}^{* T} \boldsymbol{Y}, \sigma^{2}\left(\boldsymbol{X}^{* T} \boldsymbol{X}^{*}+\boldsymbol{D}_{\boldsymbol{\tau}}{ }^{-1}\right)^{-1}\right)
$$

where $\boldsymbol{D}_{\boldsymbol{\tau}}$ is a diagonal matrix with diagonal $\left(K, K, \tau_{1}^{2}, \ldots, \tau_{p}^{2}\right)$. To speed up computation time this can be broken up into block updates instead of all $p$ variables at once.

2. For $j=1, \ldots, p$ sample $\gamma_{j}$ from a bernoulli distribution with probability

$$
\frac{\frac{\lambda_{1}}{\sigma} e^{\frac{-\lambda_{1}\left|\beta_{j}\right|}{\sigma}} \theta^{w_{j}}}{\frac{\lambda_{1}}{\sigma} e^{\frac{-\lambda_{1}\left|\beta_{j}\right|}{\sigma}} \theta^{w_{j}}+\frac{\lambda_{0}}{\sigma} e^{\frac{-\lambda_{0}\left|\beta_{j}\right|}{\sigma}}\left(1-\theta^{w_{j}}\right)}
$$

3. Use a random-walk Metropolis Hastings algorithm, rejection sampling, or any other approach to sampling from a univariate distribution without a closed form, to sample from the full conditional of $\theta$

$$
p(\theta \mid \bullet) \propto \theta^{a+\sum_{j=1}^{p} w_{j} \gamma_{j}}(1-\theta)^{b} \prod_{j=1}^{p}\left(1-\theta^{w_{j}}\right)^{\left(1-\gamma_{j}\right)}
$$

\footnotetext{
*Department of Statistics, University of Florida, 102 Griffin-Floyd Hall, P.O. Box 118545, Gainesville, Fl, 32611, USA jantonelli@ufl.edu

${ }^{\dagger}$ Department of Biostatistics, Harvard T.H. Chan School of Public Health, 677 Huntington Avenue, Boston, MA, 02115, USA fdominic@hsph.harvard.edu

${ }_{\ddagger}$ Department of Biostatistics and Computational Biology, CLS 11007, Dana-Farber Cancer Institute, 450 Brookline Ave, Boston, MA, 02215, USA gp@jimmy.harvard.edu
} 
4. Letting $\eta_{j}^{2}=1 / \tau_{j}^{2}$, sample from the full conditional:

$$
\eta_{j}^{2} \mid \bullet \sim \operatorname{InvGauss}\left(\mu^{\prime}=\sqrt{\frac{\lambda^{\prime} \sigma^{2}}{\beta_{j}^{2}}}, \lambda^{\prime}=\left(\lambda_{1} \gamma_{j}+\lambda_{0}\left(1-\gamma_{j}\right)\right)^{2}\right)
$$

where the inverse-Gaussian density is given by

$$
f(x)=\sqrt{\frac{\lambda^{\prime}}{2 \pi}} x^{-3 / 2} \exp \left\{\frac{\lambda^{\prime}\left(x-\mu^{\prime}\right)^{2}}{2 \mu^{\prime 2} x}\right\}
$$

5. Sample $\sigma^{2}$ from the full conditional

$$
\sigma^{2} \mid \bullet \sim \operatorname{InvGamma}\left(c+\frac{n}{2}+\frac{p}{2}, d+\frac{\left\|\boldsymbol{Y}-\boldsymbol{X}^{*} \boldsymbol{\beta}^{*}\right\|^{2}}{2}+\frac{\boldsymbol{\beta}^{T} \boldsymbol{D}_{\tau, \beta}^{-1} \boldsymbol{\beta}}{2}\right)
$$

Where $\boldsymbol{D}_{\tau, \beta}$ is a diagonal matrix with diagonal $\left(\tau_{1}^{2}, \ldots, \tau_{p}^{2}\right)$

\section{Finding empirical Bayes estimate of $\lambda_{0}$}

As seen in Casella (2001) the Monte Carlo EM algorithm treats the parameters as missing data and iterates between finding the "complete data" log likelihood where the parameter values are filled in with their expectations from the gibbs sampler, and maximizing this expression as a function of the hyperparameter values. After removing the terms not involving $\lambda_{0}$ the "complete data" log likelihood can be written as

$$
\left(p-\sum_{j=1}^{p} \gamma_{j}\right) \ln \left(\lambda_{0}^{2}\right)-\frac{\lambda_{0}^{2}}{2} \sum_{j=1^{p}} \tau_{j}^{2} 1\left(\gamma_{j}=1\right)
$$

And now, adopting the same notation that is typically used with the EM algorithm and was seen in Park and Casella (2008) we can take the expectation of this expression, where expectations are taken as the averages over the previous iterations posterior samples

$$
Q\left(\lambda_{0} \mid \lambda_{0}^{(k-1)}\right)=\left(p-\sum_{j=1}^{p} E_{\lambda_{0}^{(k-1)}}\left(\gamma_{j}\right)\right) \ln \left(\lambda_{0}^{2}\right)-\frac{\lambda_{0}^{2}}{2} \sum_{j=1}^{p} E_{\lambda_{0}^{(k-1)}}\left(\tau_{j}^{2} 1\left(\gamma_{j}=1\right)\right)
$$

And now we can find the maximum of this expression with respect to $\lambda_{0}$ and we find that the maximum occurs at

$$
\lambda_{0}^{(k)}=\sqrt{\frac{2\left(p-E_{\lambda_{0}^{(k-1)}}\left(\sum_{j} \gamma_{j}\right)\right)}{\sum_{j} E_{\lambda_{0}^{(k-1)}}\left(\tau_{j}^{2} 1\left(\gamma_{j}=0\right)\right)}}
$$




\section{Posterior mode estimation}

In the manuscript we briefly described the details involved with estimating the posterior mode of our model. Here we provide a more detailed description of the coordinate ascent algorithm. These ideas were first introduced in Ročková and George (2016), and we extend them here to differing weights, $w_{j}$. We will defer many of the implementation details to Ročková and George (2016), though we will highlight many of them here to illustrate how the weights, $w_{j}$ can reduce shrinkage for potential confounders.

\section{Assuming $\theta$ is fixed}

Adopting similar notation to Ročková and George (2016) we can formulate the problem in the standard penalized regression framework

$$
\left(\widehat{\beta_{0}}, \widehat{\beta_{t}}, \widehat{\boldsymbol{\beta}}\right)=\underset{\left(\beta_{0}, \beta_{t}, \boldsymbol{\beta}\right) \in \mathbb{R}^{p+2}}{\operatorname{argmax}}\left\{-\frac{1}{2}|| \boldsymbol{Y}-\boldsymbol{X} \boldsymbol{\beta}-\beta_{t} \boldsymbol{T}-\beta_{0} \|^{2}+\operatorname{pen}(\boldsymbol{\beta} \mid \theta)\right\}
$$

Notice that in the penalty we have conditioned on a value of $\theta$. To illustrate the results and the implementation of the approach we will first illustrate them for a fixed value of $\theta$. Obviously, we want to let the data inform $\theta$, which will then provide us with multiplicity control Scott et al. (2010). As shown in Ročková and George (2016) the extension to random values of $\theta$ is straightforward and the same results will apply with a minor adjustment. The penalty can be written as

$$
\begin{aligned}
\operatorname{pen}(\boldsymbol{\beta} \mid \theta) & =\sum_{j=1}^{p} \rho\left(\beta_{j} \mid \theta\right) \\
& =\sum_{j=1}^{p}-\lambda_{1}\left|\beta_{j}\right|+\log \left(\frac{p_{\theta}^{*}(0)}{p_{\theta}^{*}\left(\beta_{j}\right)}\right)
\end{aligned}
$$

where

$$
p_{\theta}^{*}\left(\beta_{j}\right)=P\left(\gamma_{j}=1 \mid \beta_{j}, \theta\right)=\frac{\theta^{w_{j}} \psi_{1}\left(\beta_{j}\right)}{\theta^{w_{j}} \psi_{1}\left(\beta_{j}\right)+\left(1-\theta^{w_{j}}\right) \psi_{0}\left(\beta_{j}\right)}
$$

An important feature of the penalty can be seen by looking at it's derivative, which gives insight into the level of shrinkage induced for $\beta_{j}$.

$$
\frac{\partial \operatorname{pen}(\boldsymbol{\beta} \mid \theta)}{\partial\left|\beta_{j}\right|} \equiv-\lambda_{\theta}^{*}\left(\beta_{j}\right)
$$


where

$$
\lambda_{\theta}^{*}\left(\beta_{j}\right)=\lambda_{1} p_{\theta}^{*}\left(\beta_{j}\right)+\lambda_{0}\left(1-p_{\theta}^{*}\left(\beta_{j}\right)\right)
$$

The intuition for $\lambda_{\theta}^{*}\left(\beta_{j}\right)$ is the larger it gets, the more shrinkage that is induced for $\beta_{j}$. If $p_{\theta}^{*}\left(\beta_{j}\right)$ is large, then it is likely that covariate $j$ is important and it will get shrunk by a smaller amount. These ideas can be seen by looking at the eventual estimation strategy for estimating $\widehat{\boldsymbol{\beta}}$, which we detail now. We can define

$$
\Delta_{j} \equiv \inf _{t>0}\{n t / 2-\rho(t \mid \theta) / t\}
$$

where there is a subscript $j$, because $\rho(t \mid \theta)$ depends on the weight, $w_{j}$. Then the global mode, $\widehat{\boldsymbol{\beta}}$ satisfies the following

$$
\widehat{\beta_{j}}=\left\{\begin{array}{lr}
0 & \text { if }\left|z_{j}\right| \leq \Delta_{j} \\
\frac{1}{n}\left(\left|z_{j}\right|-\lambda_{\theta}^{*}\left(\widehat{\beta}_{j}\right)\right)_{+} \operatorname{sign}\left(z_{j}\right) & \text { if }\left|z_{j}\right|>\Delta_{j}
\end{array}\right.
$$

where $z_{j}=\boldsymbol{X}_{\boldsymbol{j}}{ }^{\prime}\left(\boldsymbol{Y}-\sum_{l \neq j} \boldsymbol{X}_{\boldsymbol{l}} \widehat{\beta_{l}}-\boldsymbol{T} \widehat{\beta_{t}}-\widehat{\beta_{0}}\right)$. This highlights a couple very important features both for implementation of the approach as well as understanding the role of the prior distribution in penalizing covariates when the goal is confounder selection. This is very useful for implementation, as we can adopt a coordinate ascent algorithm that iterates through each of the covariates and updates them according to (3.7). We also now see the role that $p_{\theta}^{*}\left(\beta_{j}\right)$, and therefore $w_{j}$ plays in both the shrinkage of covariates and the decision to force them to zero or not. As $w_{j}$ gets smaller, $p_{\theta}^{*}\left(\beta_{j}\right)$ gets larger and then $\Delta_{j}$ gets smaller making it less likely that we estimate $\beta_{j}$ to be zero. For those coefficients that are not thresholded to zero, there still exists soft thresholding or shrinkage and this is dictated by $\lambda_{\theta}^{*}\left(\beta_{j}\right)$. The smaller we set $w_{j}$, the smaller $\lambda_{\theta}^{*}\left(\beta_{j}\right)$ becomes leading to less shrinkage. This shows that if we can set $w_{j}$ to be smaller for potential confounders then we have both increased their probability of making it into the final model (i.e $\widehat{\beta}_{j} \neq 0$ ), and we have reduced the shrinkage affecting potential confounders.

\section{Assuming $\theta$ is random}

Now that we have illustrated the approach for a fixed value of $\theta$, we can extend it to random $\theta$, by letting the data inform the level of sparsity. Ročková and George (2016) showed a crucial result when attempting to implement a random value for $\theta$. Many of the previous results were allowed because the penalty on $\boldsymbol{\beta}$ was separable, i.e the penalties for each $\beta_{j}$ were independent. Now they are not independent as the penalty marginalizes over $\theta$, which is estimated from all of the regression coefficients and the penalties are no longer marginally independent. Ročková and George (2016) 
showed, however, that all of the previous results hold in the situation where $\theta$ is estimated if we replace $\theta$ in all the expressions with $\theta_{j}=E\left(\theta \mid \widehat{\boldsymbol{\beta}}_{\backslash j}\right)$ where $\widehat{\boldsymbol{\beta}}_{\backslash j}$ is the vector of estimated regression coefficients with the $j^{\text {th }}$ element removed. When $p$ is large then $E\left(\theta \mid \widehat{\boldsymbol{\beta}}_{\backslash j}\right) \approx E(\theta \mid \widehat{\boldsymbol{\beta}})$, and therefore we can let the value of $\theta$ remain constant for each $j$. This conditional expectation is given by

$$
E(\theta \mid \widehat{\boldsymbol{\beta}})=\frac{\int_{0}^{1} \theta^{a}(1-\theta)^{b-1} \prod_{j=1}^{p}\left\{\theta^{w_{j}} \lambda_{1} e^{-\left|\widehat{\beta_{j}}\right|\left(\lambda_{1}\right)}+\left(1-\theta^{w_{j}}\right) \lambda_{0} e^{-\widehat{\beta_{j}} \mid\left(\lambda_{0}\right)}\right\} d \theta}{\int_{0}^{1} \theta^{a-1}(1-\theta)^{b-1} \prod_{j=1}^{p}\left\{\theta^{w_{j}} \lambda_{1} e^{-\left|\widehat{\beta_{j}}\right|\left(\lambda_{1}\right)}+\left(1-\theta^{w_{j}}\right) \lambda_{0} e^{-\widehat{\beta_{j}} \mid\left(\lambda_{0}\right)}\right\} d \theta}
$$

This expression does not have a closed form, but is fairly easy to calculate numerically. Because we can simply take all the implementation steps from above and replace $\theta$ with $E(\theta \mid \widehat{\boldsymbol{\beta}})$, we can update our coordinate ascent algorithm accordingly. Now after each time we update $\beta_{j}$ for each $j$, we must also update $\theta$ according to (3.8). This can be computationally expensive, particularly in very high-dimensional settings. In reality $E(\theta \mid \widehat{\boldsymbol{\beta}})$, will not change much after each updated $\beta_{j}$, especially in sparse settings when most of the parameters are not included in the model, i.e $\beta_{j}=0$. In practice, one can update $\theta$ in the coordinate ascent algorithm after every $m^{\text {th }}$ covariate is updated, where $m$ is some large value that is also smaller than $p$.

\section{Selection of $\lambda_{0}$}

As with fully Bayesian inference, our coordinate ascent algorithm relies on a well chosen value of $\lambda_{0}$, and there are two distinct solutions within this paradigm. The first of which, cross validation, is commonly used for penalized likelihood procedures to find tuning parameter values. Cross validation is used to find a value of the tuning parameter that aims to optimize the model's predictive ability. We are interested in confounding adjustment, not prediction, though cross validation still might ultimately be useful for finding an appropriate $\lambda_{0}$ estimate. Another approach, as described in Ročková and George (2016), involves estimating the model for an increasing sequence of $\lambda_{0}$ values and then finding the value of $\lambda_{0}$ at which the estimates stabilize. We can evaluate $\widehat{\boldsymbol{\beta}}$ for $\lambda_{0} \in\left\{\lambda_{0}^{(1)}, \lambda_{0}^{(2)}, \ldots\right\}$, which is a sequence of penalties that more aggressively penalize coefficients. As shown in Ročková and George (2016) the estimates $\widehat{\boldsymbol{\beta}}$ eventually converge as we increase $\lambda_{0}$ and we can take the estimates of $\boldsymbol{\beta}$ at this stabilization point as the final posterior mode estimate.

\section{Theoretical considerations}

Ročková and George (2016) provide a number of asymptotic results justifying the use of the spike and slab lasso prior in high dimensional settings. In particular, they derived asymptotic rates of convergence for $\boldsymbol{\beta}$, such as the fact that $|X(\widehat{\boldsymbol{\beta}}-\boldsymbol{\beta})|_{2}<$ $C_{1} \sqrt{q\left(1+C_{2}\right) \log p}$, where $C_{1}, C_{2}$ are positive constants and $q=\|\boldsymbol{\beta}\|_{0}$. It is a straightforward application of their proofs to obtain identical results under our prior, however, 
our interest is in estimating $\beta_{t}$, not in estimating $\boldsymbol{\beta}$. One can use Equation 3.7 to see that

$$
\left.\widehat{\beta}_{t}=\beta_{t}+\frac{1}{n}\left(\boldsymbol{T}^{\prime}(\boldsymbol{X} \boldsymbol{\beta}-\boldsymbol{X} \widehat{\boldsymbol{\beta}})+\boldsymbol{T}^{\prime} \boldsymbol{\epsilon}\right)\right)
$$

We can then apply the triangle inequalty and Hölder's inequality to see that

$$
\begin{aligned}
\left|\widehat{\beta}_{t}-\beta_{t}\right|_{1} & \leq\left|\frac{1}{n} \boldsymbol{T}^{\prime}(\boldsymbol{X} \boldsymbol{\beta}-\boldsymbol{X} \widehat{\boldsymbol{\beta}})\right|_{1}+\frac{1}{n}\left|\boldsymbol{T}^{\prime} \boldsymbol{\epsilon}\right|_{1} \\
& \leq \frac{1}{n}|\boldsymbol{T}|_{2}|(\boldsymbol{X} \boldsymbol{\beta}-\boldsymbol{X} \widehat{\boldsymbol{\beta}})|_{2}+\frac{1}{n}\left|\boldsymbol{T}^{\prime} \boldsymbol{\epsilon}\right|_{1}=O\left(\sqrt{\frac{\log p}{n}}\right)
\end{aligned}
$$

where in the last step we used that $\frac{1}{n}\left|\boldsymbol{T}^{\prime} \boldsymbol{\epsilon}\right|_{1} \rightarrow 0$ and we made the assumption that $T$ has bounded second moments, which is very reasonable, particularly when $T$ is binary where it is certain to hold. This shows that our estimate of the treatment effect converges, but at a slower rate than $n^{1 / 2}$ as we see the usual $\log p$ penalty found in high-dimensional models. Interestingly, Belloni et al. (2014); Farrell (2015); Belloni et al. (2017); Athey et al. (2016) showed that $n^{1 / 2}$-consistent estimation can be achieved when estimating treatment effects in high dimensional scenarios. Our approach is mainly targeted at improving finite sample properties instead of asymptotic rates.

\section{Simulations with different sample sizes and confounding strengths}

Here we present simulation results that build on those found in the main manuscript. We will present three different scenarios here: 1) A scenario that is the same as the one of Section 4.1, but with $n=400$ and $p=800,2)$ A scenario that is the same as the simulation in Section 4.3, but with $n=400$ and $p=800$, and 3) A scenario that is the same as the simulation from Section 4.3 where the confounding strength has been increased by setting $\|\boldsymbol{\beta}\|_{2}=18$. Below we present the corresponding tables for each of these scenarios. 


\section{Additional scenario 1:}

\begin{tabular}{lrrrr}
\hline type & \% Bias & MSE & $95 \%$ interval coverage & $E\left(\widehat{S E}\left(\widehat{\beta}_{t}\right)\right) / S D\left(\widehat{\beta}_{t}\right)$ \\
\hline Outcome LASSO & 39.6 & 0.21 & & \\
Post LASSO & 24.2 & 0.11 & & \\
Double post selection & 14.4 & 0.09 & 0.76 & 0.73 \\
Approximate residual de-biasing & 30.1 & 0.14 & 0.76 & 1.04 \\
Doubly robust lasso & 14.1 & 0.17 & 0.71 & 0.61 \\
EM-SSL & 6.9 & 0.05 & 0.93 & 0.95 \\
EM-SSL Heterogeneous & 9.5 & 0.06 & 0.93 & 1.06 \\
EM-SSL Restricted & 6.4 & 0.05 & 0.93 & 0.95 \\
EM-SSL Restricted Heterogeneous & 9.4 & 0.06 & 0.94 & 1.05 \\
\hline
\end{tabular}

Table 1: Results for estimating the average treatment effect under the first additional simulation scenario.

\section{Additional scenario 2:}

\begin{tabular}{lrrrr}
\hline type & \% Bias & MSE & $95 \%$ interval coverage & $E\left(\widehat{S E}\left(\widehat{\beta}_{t}\right)\right) / S D\left(\widehat{\beta}_{t}\right)$ \\
\hline Outcome LASSO & 0.0 & 0.36 & & \\
Post LASSO & 0.0 & 0.40 & 0.80 & 0.67 \\
Double post selection & 0.0 & 0.38 & 0.85 & 0.79 \\
Approximate residual de-biasing & 0.0 & 0.47 & 0.67 & 0.50 \\
Doubly robust lasso & 0.0 & 1.42 & 0.79 & 0.67 \\
EM-SSL & 0.0 & 0.34 & 0.93 & 0.96 \\
EM-SSL Heterogeneous & 0.0 & 0.51 & 0.94 & 1.00 \\
EM-SSL Restricted & 0.0 & 0.21 & 0.97 & 1.03 \\
EM-SSL Restricted Heterogeneous & 0.0 & 0.42 & & \\
\hline
\end{tabular}

Table 2: Results for estimating the average treatment effect under the second additional simulation scenario.

\section{Additional scenario 3:}

\begin{tabular}{lrrrr}
\hline type & \% Bias & MSE & $95 \%$ interval coverage & $E\left(\widehat{S E}\left(\widehat{\beta}_{t}\right)\right) / S D\left(\widehat{\beta}_{t}\right)$ \\
\hline Outcome LASSO & 0.0 & 14.28 & & \\
Post LASSO & 0.0 & 12.31 & & \\
Double post selection & 0.0 & 15.08 & 0.82 & 0.72 \\
Approximate residual de-biasing & 0.0 & 21.44 & 0.80 & 0.66 \\
Doubly robust lasso & 0.0 & 13.75 & 0.43 & 0.37 \\
EM-SSL & 0.0 & 12.25 & 0.82 & 0.71 \\
EM-SSL Heterogeneous & 0.0 & 24.46 & 0.89 & 0.84 \\
EM-SSL Restricted & 0.0 & 8.83 & 0.93 & 0.96 \\
EM-SSL Restricted Heterogeneous & 0.0 & 18.76 & 0.95 & 0.98 \\
\hline
\end{tabular}

Table 3: Results for estimating the average treatment effect under the third additional simulation scenario. 


\section{Simulations for sensitivity to hyperparameters}

First, we will assess the sensitivity to the choice of $\lambda_{1}$ by running the same analysis as in Section 4.1 of the manuscript and varying $\lambda_{1} \in\{0.05,0.25,0.5\}$. Below are the tables for the three different values of $\lambda_{1}$ and we see that the results are highly similar to those in the manuscript showing the robustness to the choice of this parameter.

\begin{tabular}{|c|c|c|c|c|}
\hline type & $\%$ Bias & MSE & $95 \%$ interval coverage & $E\left(\widehat{S E}\left(\widehat{\beta}_{t}\right)\right) / S D\left(\widehat{\beta}_{t}\right)$ \\
\hline EM-SSL & 15.00 & 0.11 & 0.92 & 1.00 \\
\hline EM-SSL Heterogeneous & 22.00 & 0.16 & 0.90 & 1.03 \\
\hline EM-SSL Restricted & 15.00 & 0.11 & 0.91 & 1.00 \\
\hline EM-SSL Restricted Heterogeneous & 24.00 & 0.16 & 0.88 & 1.03 \\
\hline
\end{tabular}

$$
\lambda_{1}=0.25
$$

\begin{tabular}{lrrrr}
\hline type & \% Bias & MSE & 95\% interval coverage & $E\left(\widehat{S E}\left(\widehat{\beta}_{t}\right)\right) / S D\left(\widehat{\beta}_{t}\right)$ \\
\hline EM-SSL & 14.00 & 0.10 & 0.93 & 1.01 \\
EM-SSL Heterogeneous & 24.00 & 0.16 & 0.90 & 1.04 \\
EM-SSL Restricted & 14.00 & 0.10 & 0.92 & 1.01 \\
EM-SSL Restricted Heterogeneous & 24.00 & 0.16 & 0.89 & 1.05
\end{tabular}

\begin{tabular}{lrrrr}
\multicolumn{1}{l}{$=\mathbf{0 . 5}$} & & & \\
& & & & \\
\hline type & \% Bias & MSE & $95 \%$ interval coverage & $E\left(\widehat{S E}\left(\widehat{\beta}_{t}\right)\right) / S D\left(\widehat{\beta}_{t}\right)$ \\
\hline EM-SSL & 14.00 & 0.10 & 0.93 & 1.02 \\
EM-SSL Heterogeneous & 25.00 & 0.16 & 0.89 & 1.07 \\
EM-SSL Restricted & 15.00 & 0.10 & 0.92 & 1.01 \\
EM-SSL Restricted Heterogeneous & 26.00 & 0.16 & 0.88 & 1.06
\end{tabular}

Now we also want to assess the sensitivity to the choice of $a$ and $b$ which dictate the prior distribution for $\theta$. Again we will use the same analysis as in Section 4.1 of the manuscript except we will vary the values of $a$ and $b$. In the manuscript we set $a=1$ and $b=0.1 p$. Here we will try two different scenarios: $a=1$ and $b=1$, as well as $a=1$ and $b=p$. The first of these scenarios represents a situation where the prior probability of entering into the slab is approximately 0.5 , and therefore much less sparse. The second situation is a more stringent prior that more strongly imposes sparsity in the model as it is less likely a priori to enter the slab. We see in the results below that our approach seems to be robust to the choice of these parameters as we obtain similar MSE and interval coverage values on either end of the spectrum of prior sparsity. 


\begin{tabular}{lrrrr}
$\mathbf{a}=\mathbf{1}, \mathbf{b}=\mathbf{p}$ & & & & \\
\hline type & \% Bias & MSE & $95 \%$ interval coverage & $E\left(\widehat{S E}\left(\widehat{\beta}_{t}\right)\right) / S D\left(\widehat{\beta}_{t}\right)$ \\
\hline EM-SSL & 15.00 & 0.11 & 0.92 & 1.00 \\
EM-SSL Heterogeneous & 22.00 & 0.16 & 0.90 & 1.04 \\
EM-SSL Restricted & 15.00 & 0.11 & 0.91 & 1.00 \\
EM-SSL Restricted Heterogeneous & 24.00 & 0.16 & 0.88 & 1.03
\end{tabular}

$$
a=1, b=1
$$

\begin{tabular}{lrrrr}
\hline type & \% Bias & MSE & $95 \%$ interval coverage & $E\left(\widehat{S E}\left(\widehat{\beta}_{t}\right)\right) / S D\left(\widehat{\beta}_{t}\right)$ \\
\hline EM-SSL & 14.00 & 0.10 & 0.92 & 1.00 \\
EM-SSL Heterogeneous & 22.00 & 0.16 & 0.90 & 1.03 \\
EM-SSL Restricted & 15.00 & 0.10 & 0.92 & 1.00 \\
EM-SSL Restricted Heterogeneous & 24.00 & 0.16 & 0.89 & 1.04 \\
& & & & \\
\hline
\end{tabular}

\section{References}

Athey, S., Imbens, G. W., and Wager, S. (2016). "Approximate residual balancing: De-biased inference of average treatment effects in high dimensions." arXiv preprint arXiv:1604.07125.

Belloni, A., Chernozhukov, V., Fernández-Val, I., and Hansen, C. (2017). "Program Evaluation and Causal Inference With High-Dimensional Data." Econometrica, 85(1): 233-298.

Belloni, A., Chernozhukov, V., and Hansen, C. (2014). "Inference on treatment effects after selection among high-dimensional controls." The Review of Economic Studies, 81(2): 608-650.

Casella, G. (2001). "Empirical bayes gibbs sampling." Biostatistics, 2(4): 485-500.

Farrell, M. H. (2015). "Robust inference on average treatment effects with possibly more covariates than observations." Journal of Econometrics, 189(1): 1-23.

Park, T. and Casella, G. (2008). "The bayesian lasso." Journal of the American Statistical Association, 103(482): 681-686.

Ročková, V. and George, E. I. (2016). "The spike-and-slab lasso." Journal of the American Statistical Association, (just-accepted).

Scott, J. G., Berger, J. O., et al. (2010). "Bayes and empirical-Bayes multiplicity adjustment in the variable-selection problem." The Annals of Statistics, 38(5): 2587-2619. 